\title{
A Verification Study on Methodology of Urban Development Projects Planning Considering Financial Management
}

\author{
Ritsumeikan University \\ Toukyu Real Estate Co. Ltd \\ Ritsumeikan University
}

\author{
Mamoru HARUNA ${ }^{1}$ \\ Tomohiko WATANABE ${ }^{2}$ \\ Xuepeng QIAN ${ }^{3}$
}

\begin{abstract}
An attempt is made to develop and verify the effective methodology of urban development projects planning, from the aspect of finance and administration. In the study, Kusatus City, Shiga Prefecture, is selected as the subject city for the case study. A financial simulation system has been built up, which is the analysis platform for the planning. The optimization of the study is linear multi-objective problem, with seven objective indexes correspond to the development objective the Kusatsu City, and four control variables of investment to living, transportation, culture, science and amusement, and welfare infrastructure. The hybrid model concept and time-related dynamic analysis are used in the planning evaluation for final proposal. And by the successful verification of the case study, it could be said that the planning methodology is rational and effective.
\end{abstract}

【Keywords】urban development, financial simulation system, hybrid model

\section{Background}

When development reaches its peak of a period, conflicts also concentrate to a critical content. Change is expected to release the stress and keep going.

In Japan, after long high speed development, especially in Tokyo area, the system fatigue of centralization in administration has caused a lot of problems. The global economic situation calls for the correspondence to the international society. And the release of Tokyo polar situation is good for the balance promotion of the whole nation.

Shift from centralization and top-down to decentralization requires the re-assignment of the national and local roles, independent urban development with peculiarity and assurance of the public service level.

For local cities, they should enhance its ability to promote independent from the big central cities, which means a local city must perform the management itself and aiming at self-supporting and sustainable development.

The independent and sustainable urban development involves the two promotion aspects. First is for solution of the subjects, such as correction of partial construction and development, improvement of living environment, activation of local economic condition and enhancement of charm of the city. Second is for further development, such as for related environmental problems, social welfare, and correspondence to the change of the social structure.

However the current financial conditions of the local cities are not satisfied. The reduction in subsidy from national government, together with the reduction of tax for the increasing aged population in the overall passive local economy, accounted for the reduction of the annual revenue. At the same time, substantial social welfare services, new environmental measures and other social needs increase the expenditure of the local government.

In this case, effective and efficient infrastructure construction is to be carried out for the urban development

1 Faculty of Science and Engineering (TEL: +81-77-561-2736)

2 Kansai Branch (TEL: +81-6-6337-1001)

3 Department of Civil Engineering (TEL: +81-77-561-2736) 
with limited financial resources. And in this study, an attempt is made to develop the rational planning methodology of urban development considering the process of idea finding, planning and projects implementation, mainly from the economical and administrative viewpoint.

\section{Literature Review}

There have been some studies and surveys on the public investment problems. M. Haruna and M. Takebayashi developed the management system and multi-project mathematical model ${ }^{1)}$ for the rational and feasible analysis for the construction projects implementation planning. $\mathrm{T}$. Okumura $^{2)}$ of Ritsumeikan University did the study of evaluation of investment plan of urban development and construction by using financial simulation analysis based on the system approach. In his study, the financial structure of urban units has been concluded. In T. Morishita's thesis ${ }^{3)}$ about the verification study related to administrative system and financial system for the local city synthesis promotion planning, Kusatsu City was selected as the subject city to observe the influence effect from promotion projects. T. Watanabe ${ }^{4)}$ advanced this research together with the optimization concept.

The hybrid model has been used for transportation planning by K. Yamada ${ }^{5)}$. And in this research, the hybrid concept is applied to the optimization of the proposal from the time-related dynamic viewpoints.

\section{Introduction of Kusatsu City}

Kusatsu City lies the central part of Japan, in Shiga prefecture, near the largest lake - Lake Biwa. It covers about 48.22 square meters and 12 kilometers in south-north direction and 9 kilometers in east-west direction, with Rito Chiou at east, Otsu City at south Moriyama City and Lake Biwa at north. National main transportation routes such as JR Main Tokaido line, Biwa Line, Shinkansen, Meishin Expressway and Route 1 and so on cross the Kusatsu Urban area.

Kusatsu City has a population of 113,796 until October 1, Heisei 14 . It is becoming the city which plays a central role in Shiga.

Here the city is briefly introduced from the seven aspects, which are also defined as seven objective indexes in the study.

- Population: representation of the vitality of the city; Kusatsu City is one of the few cities in Japan which has the increasing population.

- Employee persons: representation of the city employment power; There is a trend of increase of the employee persons in Kusatsu City.

- Average income: representation of the civic economical content; the average income of the city keeps waving under limited control.

- The production sum of 1 st, 2 nd, 3rd industry: representation of the activity of the industry; the agriculture of the city decays slowly; and the main industry is the 2nd industry. While the 3rd industry is developing in a high speed.

- Tax: representation of the strength of the independence of city finance. There is no obvious change about tax of the city in recent years.

And the city structure is changing toward the urban type. In the comprehensive development plan made by Kusatsu City in 1999, "Vision for Kusatsu City at 2010"6), three objectives are made for the Kusatsu urban development:

(1) To keep the population scale and develop the 2nd and 3rd industry;

(2) To increase the tax for self-supporting;

(3) To keep the level of the 1st industry though no rapid expansion is expected.

So, we arranged priority positions of the seven objective indexes according the situation mentioned above. They are listed in order of importance: population, the production sum of 2 nd industry, the production sum of 3 rd industry, the tax, the employee persons, the average income and the production sum of 1 st industry.

\section{Study Procedure}

The study starts from construction of the simulation model aiming at measuring the influence effect from infrastructure construction. This part contains two steps, 
first is to analyze the complex relationship of local activities (shown in Fig.1), which is the theory basis of mathematical formulation, and second is to formulate the simulation system.

After the simulation system complete, we follow the aim and perform the simulation experiments to grasp the characteristic effects of investment. And this stage gives the most several influential investment items according to the effect they cause to the objective items.

Then we build up the multi-objective function for the planning optimization process, setting the investment items as the control variables.

Then simulation system and planning system have been concluded. So follows the combination of the two model systems based on the hybrid concepts. That is the so-called hybrid model applied in the study.

Kusatsu city was selected in the study again as the subject city in fiscal 20 years, from 1981 to 2000 , which is divided three periods because of the time-related dynamic analysis: first is from the $1^{\text {st }}$ year to the $5^{\text {th }}$ year, second from the $6^{\text {th }}$ year to the $10^{\text {th }}$ year, and third is from $11^{\text {th }}$ year to the $20^{\text {th }}$ year. The verification is made by the comparison of the output optimal distribution and the practical distribution pattern.

\section{Research Details and Results of Each stage}

\subsection{Financial Simulation System}

\section{(1) Relationship Description}

The local activities are supported by the six sectors from urban function category: disaster prevention basis, transportation basis, living basis, industrial basis, the culture, science and amusement basis and the welfare basis. The input and output are both abstracted from these media. Also the structure (Fig.1) shows the relationship of local activities simulation of Kusatsu City.

\section{(2) Mathematical Formulation}

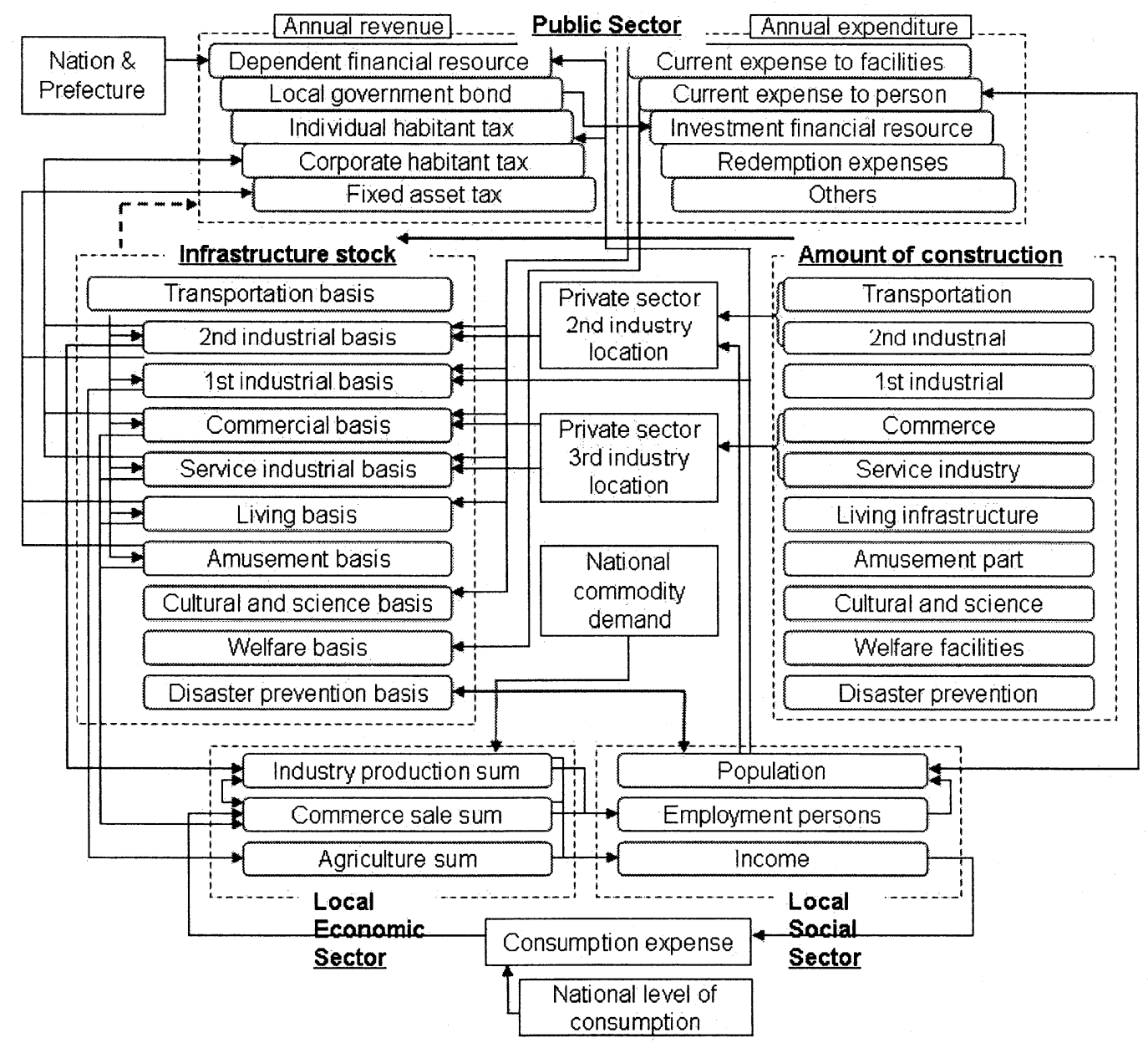

Fig.1 The Structure of Local Activities Simulation 
Based on the structure, the simulation is furthered to the mathematical formulation stage. The main functions of the financial simulation system are listed as the following:

(1) Financial flow of local government

Income:

Dependent fund (Nation subsidy + Prefecture fund + Local Tax)

$$
\begin{aligned}
F R T(t) & =\alpha_{1} L S(t-1)+\alpha_{2} W S(t-1)+\alpha_{3} S C S(t-1) \\
& +\alpha_{4} F(t-1)+C_{1}
\end{aligned}
$$

$F R T(t)$ : Total amount of dependent fund in period $\mathrm{t}$

$L S(t)$ : Living basis stock in period $t$

$W S(t)$ : Welfare basis stock in period $\mathrm{t}$

$S C S(t)$ : Culture and science basis stock in period $t$

$F(t)$ : Number of household in period $t$

The fixed asset tax

$$
\begin{aligned}
F T(t)= & \alpha_{6} 1 S(t-1)+\alpha_{7} 2 S(t-1)+\alpha_{8} 3 S(t-1) \\
& +\alpha_{9} L S(t-1)+C_{3}
\end{aligned}
$$

$F T(t)$ Total fixed asset tax in period $t$

$1 S(t)$ : 1 st industry basis stock in period $t$

$2 S(t): 2$ nd industry basis stock in period $t$

$3 S(t)$ : 3rd industry basis stock in period $t$

The individual habitant tax

$$
I H T(t)=\alpha_{10} F(t-1)+\alpha_{11} \text { AveS }(t-1)+C_{4}
$$

$I H T(t):$ the individual habitant tax in period $\mathrm{t}$ AveS $(t)$ : the average income in period $t$

The corporate habitant tax

$$
\begin{aligned}
C H T(t) & =\alpha_{12} I N(t-1)+\alpha_{13} 3 S(t-1)+\alpha_{14} \operatorname{IPS}(t) \\
& +\alpha_{15} A P S(t)+C_{5}
\end{aligned}
$$

$C H T(t)$ : the corporate habitant tax in period $t$ $\operatorname{IPS}(t)$ : the industrial production sum in period $t$ $A P S(t)$ : the agriculture production sum in period $\mathrm{t}$

Other income

$$
\operatorname{OIN}(t)=\alpha_{16} F(t-1)+\alpha_{17} \operatorname{OIN}(t-1)+C_{6}
$$

$\operatorname{OIN}(t)$ : Other income in period $\mathrm{t}$

The total income

$$
I N(t)=F T(t)+F R T(t)+I H T(t)+C H T(t)+O I N(t)
$$

$I N(t) ;$ the total income in period $\mathrm{t}$

\section{Expense:}

The current expenses to facilities

$$
\begin{aligned}
F C E(t) & =\alpha_{18} L S(t-1)+\alpha_{19} W S(t-1)+\alpha_{20} S C S(t-1) \\
& +\alpha_{21} T S(t-1)+C_{7}
\end{aligned}
$$

$F C E(t)$ : the current expenses to facilities in period $t$ $T S(t)$ : Traffic basis stock in period $\mathrm{t}$

The current expenses to the person

$P C E(t)=\alpha_{22} F(t-1)+C_{8}$

$P C E(t)$ : the current expenses to the person

The investment fiscal resource

$\operatorname{IFR}(t)=\alpha_{23} F R T(t)+\alpha_{24} F T(t)+\alpha_{25} L B(t)+C_{9}$

$\operatorname{IFR}(t)$ : the investment fiscal resource in period $\mathrm{t}$

$L B(t)$. the local government bond in period $\mathrm{t}$

The redemption expenses

$R E(t)=\alpha_{26} F T(t)+\alpha_{27} I H T(t)+\alpha_{28} T B(t-1)+C_{10}$

$R E(t)$ : the redemption expenses

$T B(t)$ the Kusatsu City bond balance remaining in an account

The Kusatsu City bond balance remaining in an account $T B(t)=\alpha_{29} T B(t-1)+\alpha_{30} R E(t)+\alpha_{31} L B(t)+C_{11}$

Other costs

$O I(t)=I N(t) \dot{\dagger}^{2} F C E(t)+P C E(t)+\operatorname{IFR}(t)+R E(t) \mathrm{i}^{2}$

$O I(t)$ Other costs in period $t$

Second, Investment to the infrastructures:

The 1st industrial basis stock

$1 S(t)=\alpha_{32} 1 I(t)+\alpha_{33} T S(t)+C_{12}$

$1 I(t)$ the 1 st industrial basis service investment in period $\mathrm{t}$

The 2nd industrial basis stock

$$
\begin{aligned}
2 S(t)= & \alpha_{34} 2 I(t-1)+\alpha_{35} 2 S(t-1)+\alpha_{36} F(t-1) \\
& +\alpha_{37} I P S(t-1)+\alpha_{38} F C E(t)+C_{13}
\end{aligned}
$$

$2 I(t)$ : the 2 nd industrial basis service investment in period $\mathrm{t}$

The 3rd industrial basis stock

$$
3 S(t)=\alpha_{39} 3 I(t)+\alpha_{40} T S(t)+\alpha_{41} F(t)+C_{14}
$$

$3 I(t)$ the 3 rd industrial basis service investment in period $\mathrm{t}$

The amusement basis stock

$$
A S(t)=\alpha_{42} A I(t-1)+\alpha_{43} T S(t)+C_{15}
$$

$A S(t)$ : the amusement stock in period $\mathrm{t}$

$A I(t)$ the amusement basis service investment in period $\mathrm{t}$

The traffic basis stock

$T S(t)=\alpha_{44} T I(t)+\alpha_{45} T S(t-1)+C_{16}$

$T I(t)$ : the traffic basis service investment in period $\mathbf{t}$ 
The living basis stock

$$
L S(t)=\alpha_{46} L I(t)+\alpha_{47} F(t)+C_{17}
$$

$L I(t)$ : the living basis service investment in period $t$ The cultural basis stock

$$
S C S(t)=\alpha_{48} S C I(t)+\alpha_{49} S C S(t-1)+C_{18}
$$

$S C I(t)$, the culture and science basis service investment in period $t$

The welfare basis stock

$$
W S(t)=\alpha_{50} W I(t)+C_{19}
$$

$W I(t)$ : the welfare basis service investment in period $\mathrm{t}$

Third, Regional economic functions:

The agriculture production sum

$$
A P S(t)=\alpha_{51} A P I(t)+\alpha_{52} 1 S(t)+\alpha_{53} F C E(t)+C_{20}
$$

$A P I(t)$ : the agriculture fault production index

The industry production sum

$$
I P S(t)=\alpha_{54} I P I(t)+\alpha_{55} C O(t)+\alpha_{56} 2 S(t)+C_{21}
$$

$I P I(t)$ : the industry production index

The annual commerce sale sum

$$
C S S(t)=\alpha_{57} 3 S(t)+\alpha_{58} C O(t)+C_{22}
$$

$\operatorname{CSS}(t)$ : the annual commerce sale sum in period $t$

The year consumption outlay

$$
C O(t)=\alpha_{59} A v e S(t)+C_{23}
$$

$C O(t)$ : the year consumption outlay in period $t$

(4) Regional social elements

The population

$$
P(t)=\alpha_{60} L S(t-1)+\alpha_{61} 3 S(t-1)+C_{24}
$$

The number of the family

$$
F(t)=\alpha_{62} P(t)+C_{25}
$$

The number of the 1 st industrial employees

$$
1 E(t)=\alpha_{63} 1 S(t)+C_{26}
$$

The number of the 2 nd industrial employees

$$
2 E(t)=\alpha_{64} I P S(t-1)+C_{27}
$$

The number of the $3 \mathrm{rd}$ industrial employees

$$
3 E(t)=\alpha_{65} 3 S(t-1)+\alpha_{66} \operatorname{CSS}(t-1)+C_{28}
$$

\section{(3) Evaluation of Simulation}

When the functions are prepared for all the relationships, the statistic data ${ }^{7)}$ of Kusatsu City of the study years are used for parameters setting, through the method of least square. Then we can reproduce the local activities and evaluate whether the simulation model is accurate or not by comparing the simulated data and real data.

We have the following figures (Fig.2, Fig.3, Fig.4, Fig.5 and Fig.6) to show that the simulation system works fairly well from the comparison of the individual habitant tax, the corporate habitant tax, the fixed asset tax, the total population and the total income of Kusatsu city from Year 1982 to Year 2000. The average tolerances of each item are also listed in Table 1.

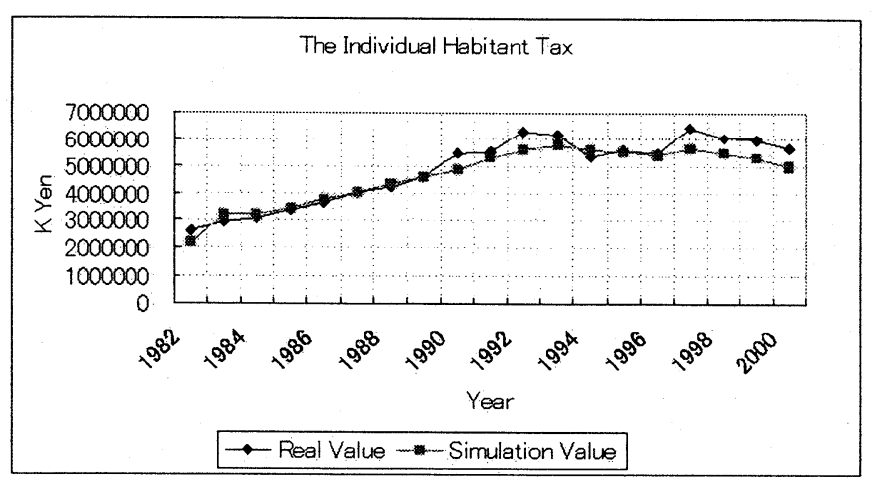

Fig.2 The Individual Habitant Tax

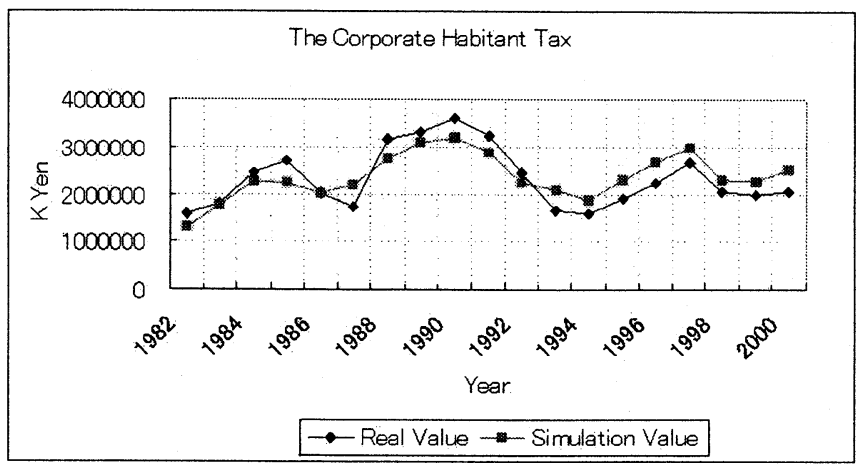

Fig.3 The Corporate Habitant

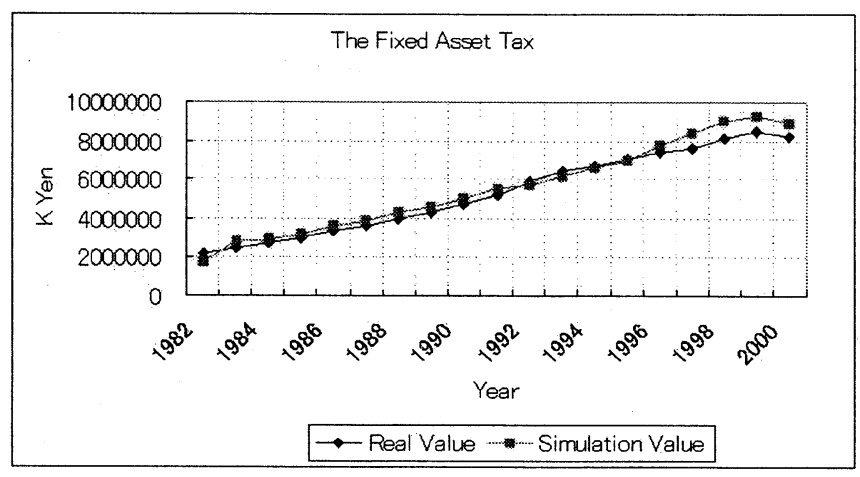

Fig.4 The Fixed Asset Tax 


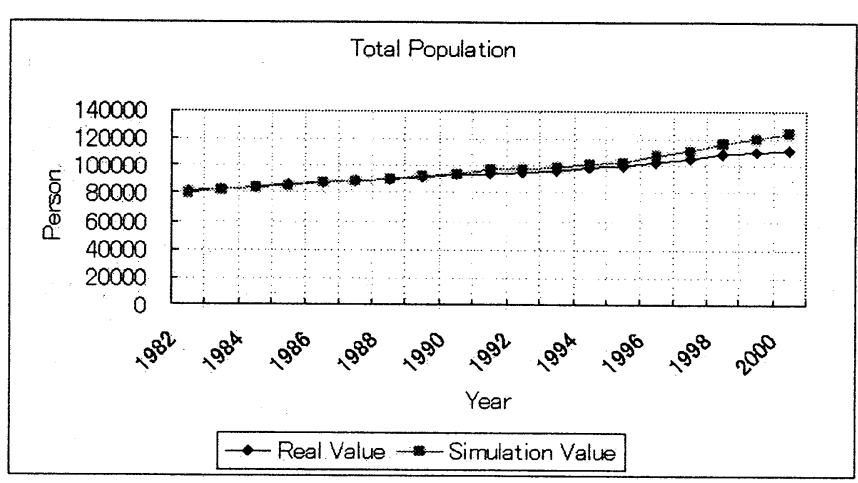

Fig.5 Total Population

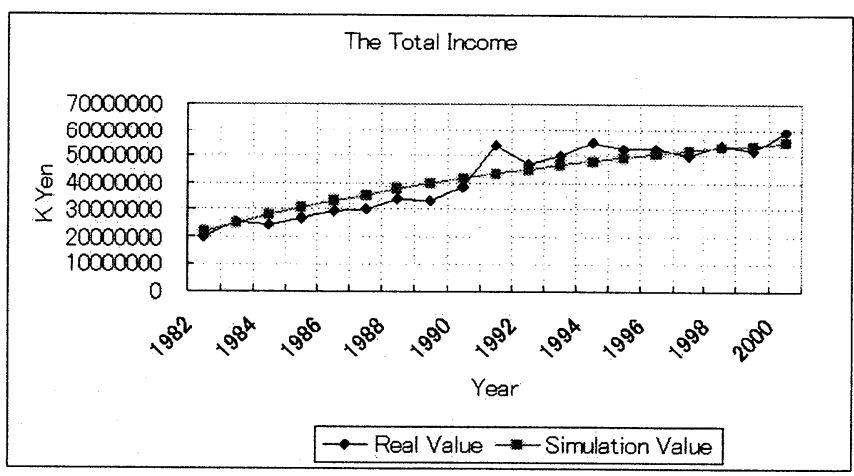

Fig. 6 The Total Income

Table 1 The Average Tolerances

\begin{tabular}{|l|r|}
\hline \multicolumn{1}{|c|}{ Item } & $\begin{array}{l}\text { Average } \\
\text { Tolerance } \\
(\%)\end{array}$ \\
\hline The individual habitant tax & 3.65 \\
\hline The corporate habitant tax & 4.05 \\
\hline The fixed asset tax & 4.46 \\
\hline The total income & 2.88 \\
\hline The total population & 2.76 \\
\hline The number of the family & 4.40 \\
\hline The employee person & 0.70 \\
\hline The agriculture production sum & 1.13 \\
\hline The industry production sum & 4.32 \\
\hline The annual commerce sale sum & 4.39 \\
\hline
\end{tabular}

\subsection{Simulation Experiments}

As we can see from the simulation system, the investment to the six kinds of infrastructure will influence the output such as population, average income, the production sum of the three industries and the tax, which are all considered as evaluation critical indexes. Then total we considered four kinds of investments, which are investment to living basis, investment to transportation basis, investment to culture and science basis and investment to welfare basis, as the four control variables, because they absorb large part of investment from government. Then among them, which one influences the indexed most and the order of them according to the influence effect are desirable for correct planning model. The experiments have been done for this aim.

There are different levels set to the content of satisfaction. According to experimental plan method, three levels and the four kinds of investments are chosen to construct $27\left(3^{4}\right)$ patterns as input to the simulation system. The software added in Microsoft Excel helped us to get the results quickly, which were then analyzed by dispersing method for the order of relationship. The proportions of distribution are shown in Table 2.

Table 2 The Patterns of Simulation Experiments

\begin{tabular}{|c|c|c|c|c|}
\hline \multicolumn{2}{|c|}{ Name of basis } & \multicolumn{3}{|c|}{ Level (\%) } \\
\hline & & 1 & 2 & 3 \\
\hline \multirow{4}{*}{ 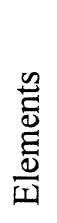 } & Living & 54 & 46 & 41 \\
\hline & Transportation & 34 & 28 & 26 \\
\hline & Culture and science & 16 & 13 & 9 \\
\hline & Welfare & 14 & 13 & 11 \\
\hline
\end{tabular}

Table 3 The Order of Relationship

\begin{tabular}{|c|c|c|c|c|c|}
\hline & & \multicolumn{4}{|c|}{ The investment to } \\
\hline & & 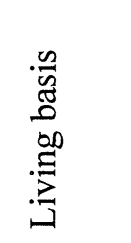 & 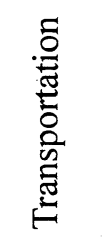 & 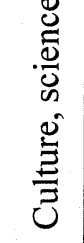 & 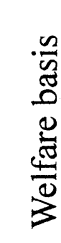 \\
\hline & Population & $\square \square \square \square$ & $\square \square \square$ & $\square \square$ & $\square$ \\
\hline \multirow{3}{*}{ 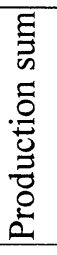 } & The 1st industry & $\square \square \square \square$ & $\square \square \square$ & $\square \square$ & $\square$ \\
\hline & The 2nd industry & $\square \square$ & $\square$ & & \\
\hline & The 3rd industry & $\square \square \square$ & $\square \square$ & $\square$ & \\
\hline & $\begin{array}{r}\text { The number of } \\
\text { employees }\end{array}$ & $\square \square \square$ & $\square \square$ & $\square$ & \\
\hline & The average income & $\square \square \square$ & $\square \square$ & $\square$ & \\
\hline & Tax & $\square \square \square$ & $\square \square$ & $\square$ & \\
\hline
\end{tabular}




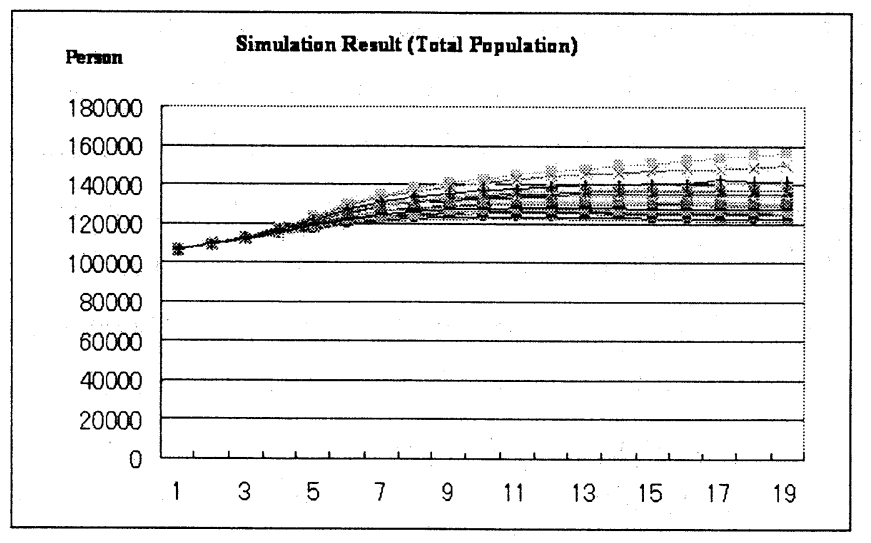

Fig.7 Simulation Result (Total Population)

(Each Line stands for one simulation pattern, totally there are 27 lines in each figure.)

Fig.7 is just one result item of total population. And Table 3 shows that the investment to the living basis will influence the seven indexed the most, and then followed by the investment to transportation basis, to culture science and amusement basis and the welfare basis in order.

\subsection{Multi-objective Function}

The multi-objective problem is not very difficult because it is made up of linear functions. Since the unit for each objective is different. We could be able to use the transition for uniform unit such as from the aspect of utility, which is one of the general methods for multi-objective problems. But here we consider the optimization from the satisfaction extent, which was developed by economist H. Simon ${ }^{8)}$. The objective functions are the seven distances to preset objective values of the seven items. And the four control variables mentioned above get parameters from the simulation stage regarding the order of influence effects.

Objective Function:

$\begin{array}{lllllll}\min & y_{p}^{-}, & \min y_{1}^{-}, & \min y_{2}^{-}, & \min y_{3}^{-}, \\ \min & y_{w}^{-}, & \min y_{i n}^{-}, & \min y_{p i}^{-}\end{array}$

Constraints:

$$
\begin{aligned}
& \alpha_{p 1} I_{l}+\alpha_{p 2} I_{r}+\alpha_{p 3} I_{c}+\alpha_{p 4} I_{w}+d_{p}-y_{p}^{+}+y_{p}^{-}=A_{p} \\
& \alpha_{11} I_{l}+\alpha_{12} I_{r}+\alpha_{13} I_{c}+\alpha_{14} I_{w}+d_{1}-y_{1}^{+}+y_{1}^{-}=A_{1} \\
& \alpha_{21} I_{l}+\alpha_{22} I_{r}+d_{2}-y_{2}^{+}+y_{2}^{-}=A_{2} \\
& \alpha_{31} I_{l}+\alpha_{32} I_{r}+\alpha_{33} I_{c}+d_{3}-y_{3}^{+}+y_{3}^{-}=A_{3} \\
& \alpha_{w 1} I_{l}+\alpha_{w 2} I_{r}+\alpha_{w 3} I_{c}+d_{w}-y_{w}^{+}+y_{w}^{-}=A_{w} \\
& \alpha_{i n 1} I_{l}+\alpha_{i n 2} I_{r}+\alpha_{i n 3} I_{c}+d_{i n}-y_{i n}^{+}+y_{i n}^{-}=A_{i n} \\
& \alpha_{p i 1} I_{l}+\alpha_{p i 2} I_{r}+\alpha_{p i 3} I_{p i}+d_{p i}-y_{p i}^{+}+y_{p i}^{-}=A_{p i}
\end{aligned}
$$

$y_{p}^{+} \times y_{p}^{-}=0, y_{1}^{+} \times y_{1}^{-}=0, y_{2}^{+} \times y_{2}^{-}=0, y_{3}^{+} \times y_{3}^{-}=0$

$y_{w}^{+} \times y_{w}^{-}=0, y_{i n}^{+} \times y_{i n}^{-}=0, y_{p i}^{+} \times y_{p i}^{-}=0$

$y_{p}^{+}, y_{p}^{-}, y_{1}^{+}, y_{1}^{-}, y_{2}^{+}, y_{2}^{-}, y_{3}^{+}, y_{3}^{-} \geq 0$

$y_{w}^{+}, y_{w}^{-}, y_{i n}^{+}, y_{i n}^{-}, y_{p i}^{+}, y_{p i}^{-} \geq 0$

$I_{l}+I_{r}+I_{c}+I_{w}=I$

$I_{l} \geq I_{l}^{*}, I_{r} \geq I_{r}^{*}, I_{c} \geq I_{c}^{*}, I_{w} \geq I_{w}^{*}$

$y_{p}^{+}, y_{p}^{-}:$Distance to objective of Population

$y_{1}^{+}, y_{1}^{-}$: Distance to objective of the 1 st industry

$y_{2}^{+}, y_{2}^{-}$: Distance to objective of the 2 nd industry

$y_{3}^{+}, y_{3}^{-}$: Distance to objective of the 3 rd industry

$y_{w}^{+}, y_{w}^{-}$: Distance to objective of employee person

$y_{i n}^{+}, y_{i n}^{-}:$Distance to objective of the average income

$y_{p i}^{+}, y_{p i}^{-}$:Distance to objective of the tax

$A_{p}$ : Objective value of Population in $\mathrm{t}$

$A_{1}$ : Objective value of the 1 st industry in $\mathrm{t}$

$A_{2}$ : Objective value of the 2 nd industry in $\mathrm{t}$

$A_{3}$ : Objective value of the $3 \mathrm{rd}$ industry in $\mathrm{t}$

$A_{w}$ : Objective value of employee persons in $\mathrm{t}$

$A_{\text {in }}:$ Objective value of the average income in $\mathrm{t}$

$A_{p i}$ : Objective value of the tax in $\mathrm{t}$

$I_{l}$ : The living basis service investment

$I_{r}$ : The traffic basis service investment

$I_{c}$ : The culture, science basis service investment

$I_{w}$ : The welfare basis service investment

The $\alpha$ used above is the coefficient of each investment item. $\Lambda$ nd the $d$ is the constant parameter of each function.

And according to the order of importance of the seven objective indexes, the minimization will be carried out by double simplex method. The constraints are designed to ensure the feasibility of the proposals.

And for the case study of Kusatsu City, the order of importance has been decided:

$$
\begin{aligned}
& \min y_{p}^{-} \gg \min y_{2}^{-}, \min y_{3}^{-} \gg>\min y_{p i}^{-}>>\min y_{p i}^{-} \\
& >>\min y_{w}^{-} \gg>\min y_{i n}^{-} \gg>\min y_{1}^{-}
\end{aligned}
$$

The production sums of 2 nd and 3 rd industries both belong to the same level. 


\subsection{Hybrid Model}

In the study, we combine simulation model and the plan model as the image expressed in Fig.8. The analysis process involves time-related dynamic concepts. From initial conditions, the simulation model provides the basic system information for the plan model, which means the parameters of the multi-objective functions. Then by solving the optimization problem, the decision 1 is concluded, which will cause a serial of change to the initial conditions. So it is necessary to perform the simulation again to update the system information. This is one cycle. Then as mentioned before, we have three terms in the study. After the first term (cycle), the analysis is continued to the second term (Year 6th to Year 10th). And updated parameters in optimization functions come together with the new plan, which is decision 2. Following the same process, we did the three cycles divided by three terms.

Fig.9, 10, 11, 12 shows the run time of the process, which is the application of decision 2 in simulation parts. And the dispersing analysis of these simulations gives the parameters for the third optimization functions.

\subsection{Verification by Case study}

In the study, related data from Kusatsu City were collected a lot. Table 4 lists the distribution patterns concluded from the three terms. And Fig.13, 14, 15, 16, 17, 18,19 show shows the comparison with the distribution patterns performed now by the local government.

The two distribution patterns were calculated from the same initial conditions and in the same financial simulation systems. And there is an average about $6 \%$ improvement of the influence effect after applying the optimal distribution plan we got from the hybrid model, from the least $1.5 \%$ to the largest $14 \%$. It can be said that the planning methodology developed in this study is rational and effective.

\section{Conclusion}

In this research, local financial activity simulation model was developed at the beginning stage of this research. Mostly through linear mathematical formulations, the simulation model analyzed the whole research period by three parts respectively. It has been proved to work well. Based on the platform of the proper simulation financial system, we carried two stages of research. In the first stage, simulation experiments were performed to measure the influence effect from infrastructure construction. As the result, four main kinds of investment were chosen as control variable for the next optimization stage. The second stage, the optimization model was also supported by the simulation model, which considered the examination of acquisition in advance of the infrastructure improvement effect. So the proposal incorporated the examination of acquisition-in-advance about the effect, which we were able to calculate in simulation system.

Positive examination, which made Kusatsu City as the object city, showed the validity of the research system with the conclusion of one effective and efficient investment pattern, which was compared with the practical one.

Regarded to optimal principle, the research aimed to the current problems and development policies and decided the importance order of the seven objectives, which were not all independent. This was from one viewpoint of optimization and could be not sufficient. In the further study, we could discuss the optimal proposal from other viewpoints. We are planning to set independent objectives, by classifying and integrating objectives of the same categories.

For the future study along this direction or methodology, we will focus on mainly two points: firstly, as the practical situation changes, we shall modify the financial simulation model. Secondly, we can apply more efficient optimization models, which have been promoted a lot, even develop new optimization method with the concepts of ideas from other fields, to the same problem we discussed here. And about the second point, we have finished the model by min-max method, and the result was fairly good according to what we have got until now.

For more wide use of the planning methodology, we are going to analyze Otsu City, which is very near to Kusatsu City in Shiga Prefecture, by the same research method. This financial analysis method can also be incorporated in other comprehensive planning projects. 


\section{APPENDIX A}

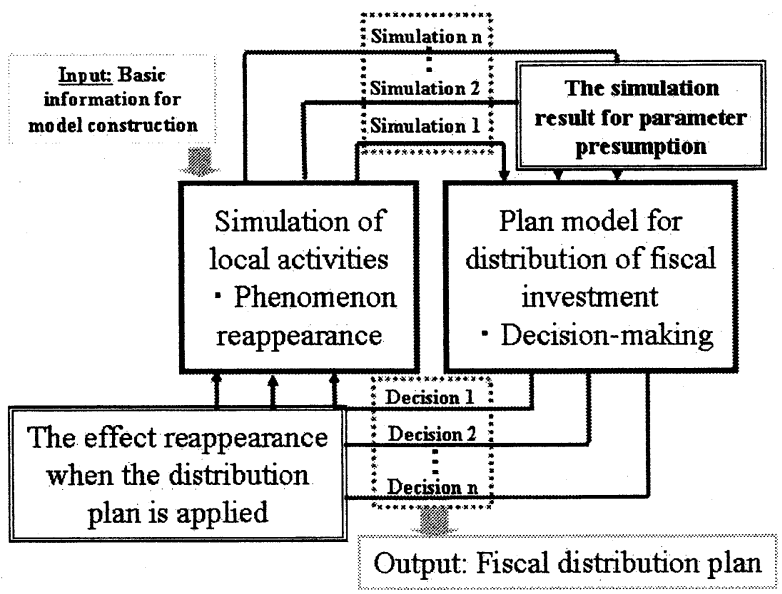

Fig.8 Hybrid Model

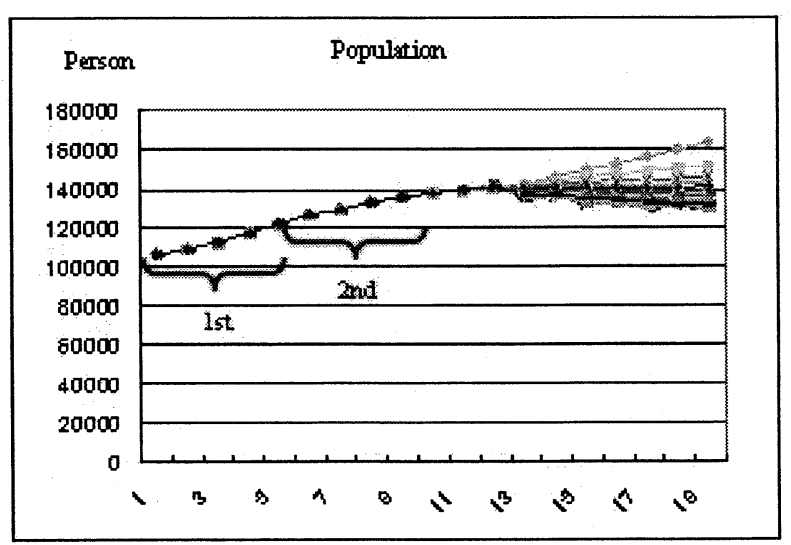

Fig.9 the 2nd phase Population Simulation (Each Line stands for one simulation pattern, totally there are 27 lines in this figure.)

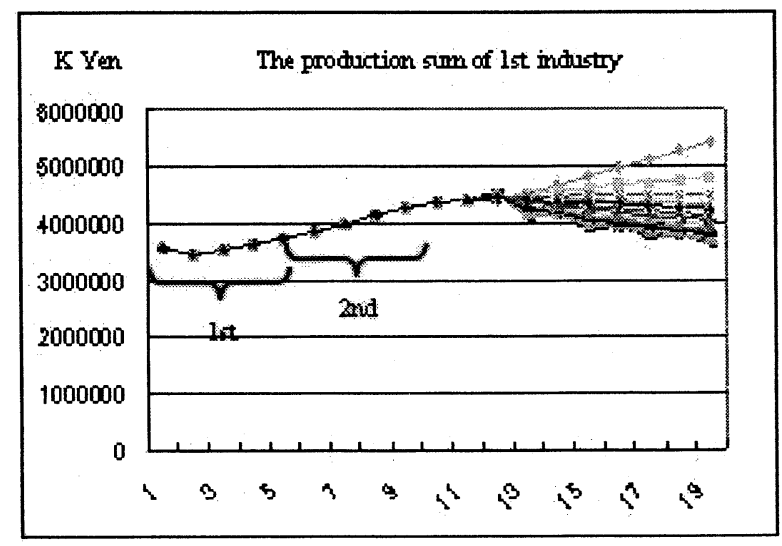

Fig.10 the 2nd phase Simulation of the Production Sum of 1st Industry (Each Line stands for one simulation pattern, totally there are 27 lines in this figure.)

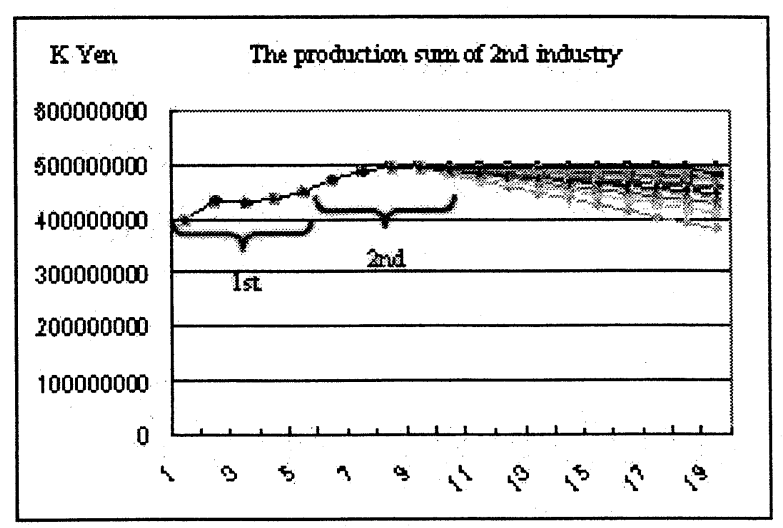

Fig.11 the 2nd phase Simulation of the Production Sum of 2nd Industry (Each Line stands for one simulation pattern, totally there are 27 lines in this figure.)

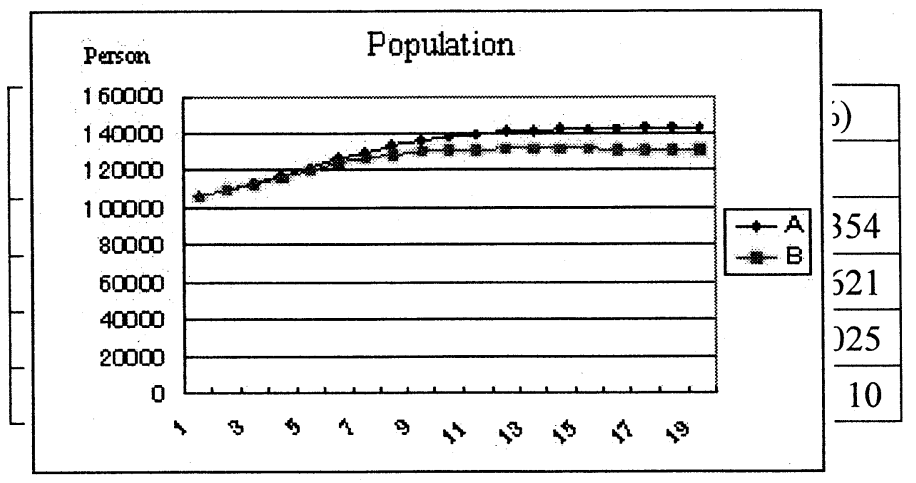

Fig.12 Final result of Population (A: the result after applying the optimal distribution from plan model; B: the result of the distribution performed now by local government, the same to Fig.13, 14, 15, 16, 17, 18)

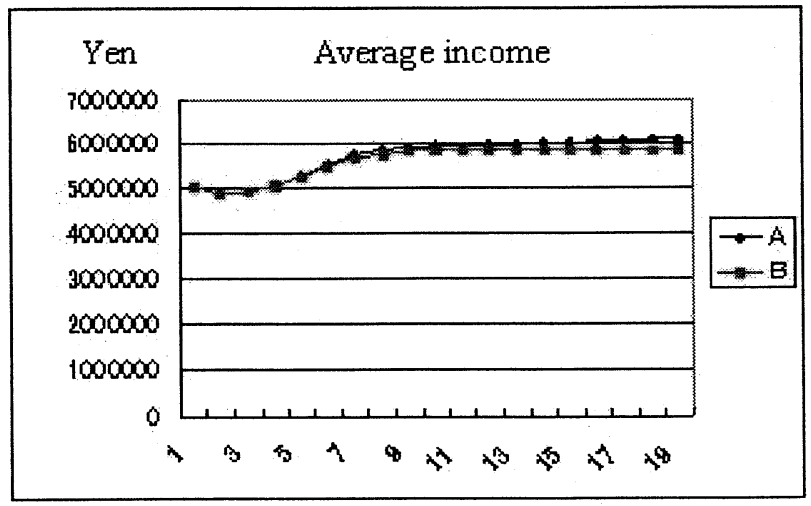

Fig.13 Final result of Population 


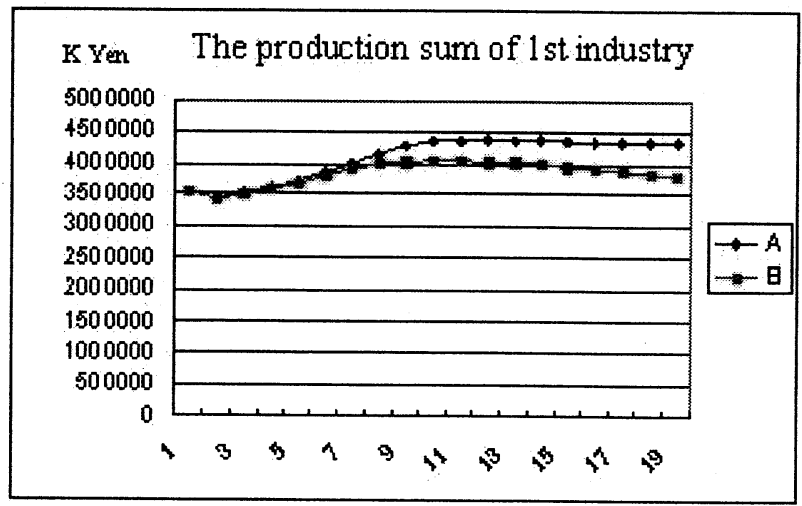

Fig.14 Final result of the production sum of 1st industry

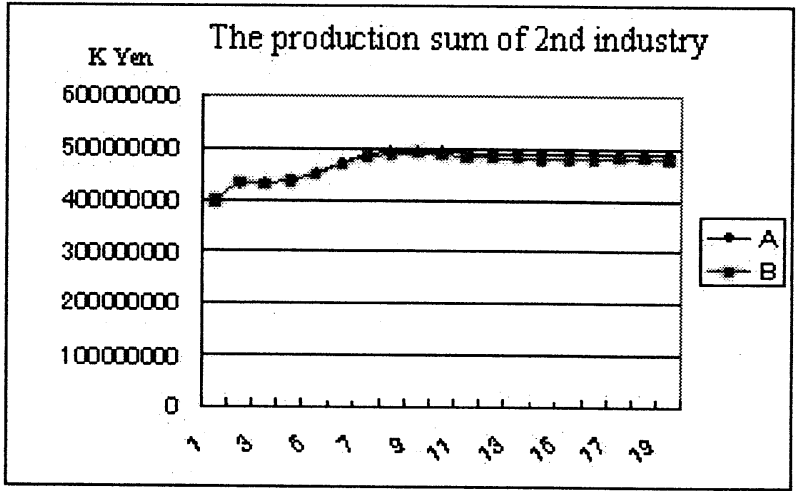

Fig.15 Final result of the production sum of 2 nd industry

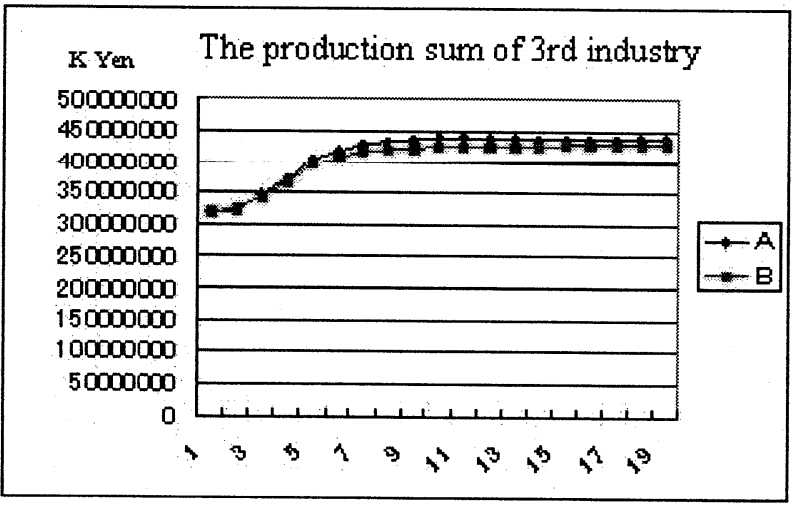

Fig.16 Final result of the production sum of 3rd industry

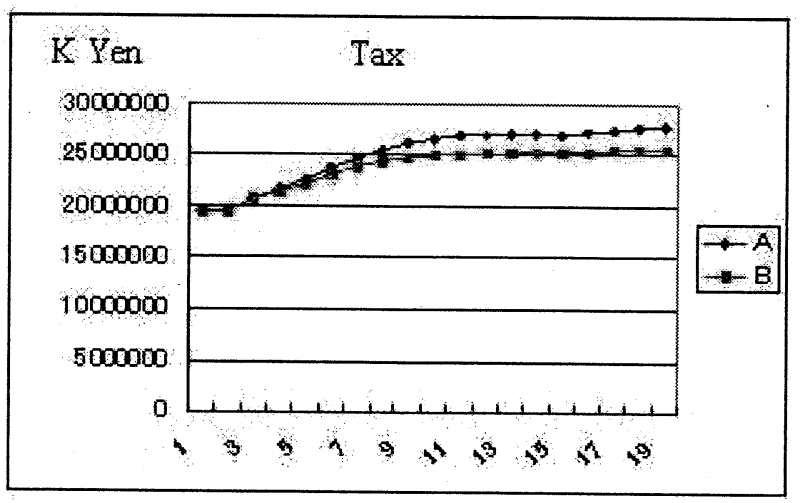

Fig.17 Final result of tax

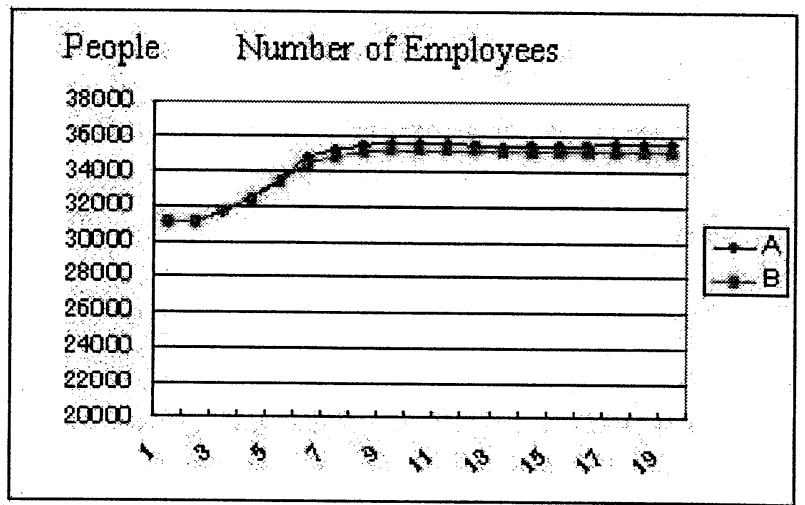

Fig.18 Final result of number of employees

\section{REFERENCES}

1) Mamoru Haruna and Mikio Takebayashi: An Optimal Programming Model for Multi-Project Planning of Regional Development and Its Application to Practical Regional Development, Studies in Regional Science, vol.28, no.1, 1998

2) Takayuki Okumura: Study on the construction of financial simulation system for the verification evaluation of local development, Master Thesis of Ritsumeikan University, 1999

3) Takeshi Morishita: Verified Study on the Urban Management of Forwarding Comprehensive Development Plan of Local City and Encouraging Proper Industries with the Application of Financial Simulation System, Master Thesis of Ritsumeikan University, 2002

4) Tomohiko Watanabe: Research on System Theory of Effective and Efficient Financial Planning Related to the Urban Construction, Master Thesis of Ritsumeikan University, 2003

5) Mamoru Haruna, Mikio Takebayashi, and Koichirou Yamada: A Study on Systems Approach to Transportation Management Problem for Desirable Urban Development Applying Hybrid Planning Model Analysis - Case Study in Otsu City of Shiga Prefecture, Proceedings of Infrastructure Planning JSCE, p.305-308, 2000

6) Kusatsu City: Comprehensive Development Plan of Kusatsu City "Vision of Kusatsu City at 2010", 1999

7) Kusatsu City: Kusatsu Annual Statistics, 1980 2001

8) Herbert Simon: Decision-Making as an Economic Resource, New Horizons of Economic Progress, 1965

9) Mamoru Haruna: The Planning Theory for Urban Development Plan and The Concept of Urban and Regional Management, Research Meeting held by Kansai Branch of JSCE, 1994 